\title{
Limits on Symmetry-Breaking Electromagnetic Interactions of the Electron and Proton.
}

\author{
B. Margolis and D. Robertson \\ Institute of Theoretical Physics, McGill University - Montreal \\ (Nuovo Cimento, 51 A, $736(1967)$ )
}

Equation (1): $f_{c} k_{\mu}$ should be $f_{c} \gamma_{\mu}$.

p. 737, line 17: $(\lambda \cdot q) q_{\mu}$ should be $(\gamma \cdot q) q_{\mu}$.

Equation (3): the denominator should read $\frac{\mathrm{d} \sigma}{\mathrm{d} \Omega}(\uparrow)+\frac{\mathrm{d} \sigma}{\mathrm{d} \Omega} \downarrow$.

Equation $(8 b): \frac{G_{E}-G_{M}}{2(1+\tau)}$ should be $\frac{G_{E}^{2}-G_{M}^{2}}{2(1+\tau)}$.

Equation (12): $f_{E}^{2}$ should be $F_{E}^{2}$.

Equation (14): $r_{2}^{0}$ should be $r_{0}^{2}$

also the square bracket ] after $E^{\prime 2} \vec{p}_{1} \cdot \bar{S}_{1} \times \bar{S}_{2}$ should be deleted and a similar bracket placed after $\frac{q^{2}}{2} \bar{P}_{2} \cdot \bar{S}_{1} \times \bar{S}_{2}$. 